adverse drug events. Further study of the consequences of different types of polypharmacy should take into account these educational differences.

\section{P51 LIFE COURSE SOCIOECONOMIC POSITION EFFECTS ON INFLAMMATION IN OLDER ADULTS: COMPENSATING FOR MISSING DATA IN THE ENGLISH LONGITUDINAL STUDY OF AGEING}

G Chatzi* ${ }^{*}$ T Chandola, A Cernat, N Shlomo. Social Statistics, University of Manchester, Manchester, UK

\subsection{6/jech-2017-SSMAbstracts. 152}

Background Greater cumulative life-course exposure to socioeconomic disadvantage is associated with higher levels of inflammatory biomarkers, C-Reactive protein and fibrinogen which are associated with increased cardiovascular disease risk. However, in most existing studies, researchers often use complete case data for analyses and ignore the impact of missing data on inference for blood based biomarker data. The English Longitudinal Study of Ageing (ELSA) has considerable missing data but the impact of missingness on inference is seldom examined.

This paper aims primarily to examine whether the levels of adulthood inflammatory biomarkers of C-Reactive protein and fibrinogen can be explained by life course socioeconomic position. We evaluate the typologies of missing data under Missing Completely at Random and Missing at Random mechanisms and methods for compensating for missing data under these mechanisms.

Methods This paper uses cross-sectional data from Wave 2 of ELSA (2004) which includes 9432 men and women aged over 52 living in England. However, only 6000 people had data for C-Reactive protein and fibrinogen. Logistic Regression modelling is implemented to identify predictors of missingness in ELSA health examination and blood collection. We use multiple linear regression modelling for analysing the association between socioeconomic position and C-Reactive protein and fibrinogen after accounting for different mechanisms of non-response using the following methods: complete case analysis (listwise deletion), inverse probability weighting and multiple imputations.

Results Participants who refused to respond to the health examination were more likely to be renters, White, single, living in London or with poor assessed health. Those who refused to give a blood sample were more likely to be older female, living East of England or London, had cancer/cardiovascular disease/stroke and poorer self-assessed health. Complete case analysis showed that people with lower education level $[0.14(\mathrm{CI} 0.04-0.25)$ and working in lower supervisory position [0.17(CI0.04-0.30)] were more likely to have higher C-Reactive protein levels. While people in the highest wealth quintile were less likely to have higher C-Reactive protein $[-0.26(\mathrm{CI}-0.36,-0.14)]$ and fibrinogen levels [-0.02(CI-0.04,$0.004)]$. These associations remained similar in inverse probability weighting and multiple imputations although there was some variation in the estimates from the different methods for compensating for missing data.

Conclusion While associations between socioeconomic position and inflammatory markers were similar across different approaches for compensating for missing data, there were differences in the estimated coefficients suggesting that it is important to account for missing biomarker data for statistical inference.

\section{P52 EARLY LIFE FACTORS ASSOCIATED WITH LIFE COURSE TRAJECTORIES OF RESTING HEART RATE}

CJ O'Hare*, D Kuh, R Hardy. MRC Unit for Lifelong Health and Ageing, University College London, London, UK

10.1136/jech-2017-SSMAbstracts. 153

Background Elevated Resting Heart Rate (RHR) is an established cardiovascular risk factor and higher RHR even in childhood has been associated with higher adult mortality rates. We are the first to describe trajectories of age-related changes in RHR in the same individuals followed from childhood to later life. We hypothesised that indicators of better health, development and socioeconomic advantage in early childhood would be reflected in lower RHR trajectories, independent of smoking status.

Methods Data were from the Medical Research Council National Survey of Health and Development (maximum $\mathrm{n}=4779$ ) followed from birth in 1946. RHR was measured on eight occasions between 6-69 years (three in childhood and five in adulthood). Information on early life factors was ascertained prospectively. Multilevel models were used to estimate life course mean RHR trajectory by sex, and to investigate associations with childhood socioeconomic position (SEP), birth weight (BW), growth from 2-6 years (conditional change in Body Mass Index (BMI) and height), duration of breast feeding (BF), and markers of neurodevelopment (age at first walking independently and cognitive ability at eight years).

Results Mean estimated RHR decreased with increasing age and plateaued from early adulthood tending to remain higher in women. In sex-adjusted analyses higher BW and conditional BMI change were associated with lower RHR at 6 years and across the life course $(-0.57 \mathrm{bpm}(95 \%$ confidence interval: $-0.95 ;-0.18)$ per $1 \mathrm{~kg}$ higher $\mathrm{BW}$ and $-0.23 \mathrm{bpm}$ $(-0.42 ;-0.05)$ per $1 \mathrm{~kg} / \mathrm{m}^{2}$ greater change in BMI); however neither influenced the rate of change with age in RHR. Neither SEP nor BF duration was associated with childhood RHR but associations with RHR trajectory emerged in adulthood such that by 60 years RHR was $1.28 \mathrm{bpm}(0.10 ; 2.16)$ higher in participants from a disadvantaged versus advantaged SEP and $-1.13 \mathrm{bpm}(-2.01 ;-0.24)$ lower for $\mathrm{BF}>=8$ months versus never. A later age at walking independently was associated with a higher RHR at 6 years $(1.33 \mathrm{bpm}(0.22 ; 2.44)$ for $>=18$ months versus $<12$ months) but lower RHR in adulthood $(-1.09 \mathrm{bpm}(-2.48 ; 0.30)$ at 60 years). Childhood cognitive ability was not associated with RHR. In a model including all early life factors, estimates were typically only slightly attenuated from sex-adjusted models except that adjustment for conditional growth substantially reduced the effect of BW. Associations were independent of life course smoking status.

Conclusion Early life is a key period in determining future RHR trajectories with effects of potentially modifiable factors persisting into the seventh decade of life. 